ORIGINAL

\title{
Presión intraabdominal y torácica en pacientes críticos con sospecha de hipertensión intraabdominal
}

\author{
F. Ruiz Ferrón*, A. Tejero Pedregosa, M. Ruiz García, A. Ferrezuelo Mata, \\ J. Pérez Valenzuela, R. Quirós Barrera y L. Rucabado Aguilar
}

Servicio de Cuidados Críticos y Urgencias, Unidad de Medicina Intensiva, Hospital Médico-Quirúrgico, Complejo Hospitalario de Jaén, Jaén, España

Recibido el 10 de junio de 2009; aceptado el 10 de febrero de 2011

Disponible en Internet el 15 de abril de 2011

\author{
PALABRAS CLAVE \\ Presión \\ intraabdominal; \\ Distensibilidad \\ torácica; \\ Presión esofágica; \\ Ventilación \\ mecánica; \\ Distensibilidad \\ abdominal
}

\begin{abstract}
Resumen
Objetivo: Analizar la correlación entre la presión intraabdominal e intratorácica en pacientes con sospecha de hipertensión intraabdominal (HIA).

Diseño: Estudio prospectivo observacional de una cohorte.

Ámbito: Unidad de medicina intensiva polivalente de un hospital universitario.

Pacientes: Se incluyó a 27 pacientes medicoquirúrgicos dependientes de ventilación mecánica controlada por fallo respiratorio agudo y con factores de riesgo de hipertensión intraabdominal. Principales variables: Medimos las presiones intraabdominal (PIA), esofágica (Peso) y de la vía aérea en condiciones estáticas (est) y dinámicas (din). Calculamos la distensibilidad del sistema respiratorio (Csr), pulmón (Cp) y pared torácica (Cpt).

Resultados: En 10 pacientes la PIAest fue mayor de $12 \mathrm{mmHg}$ (HIA, PIAest, $14 \pm 2$ [12-21] mmHg) $y$ en el resto fue normal $(n=17$; PIAest, $8 \pm 2$ [3-11] mmHg). La Pesoest fue $11 \pm 5$ (2-27) y Pesodin, $7 \pm 4(2-24) \mathrm{cmH}_{2} \mathrm{O}$. Considerando la presencia o no de $\mathrm{HIA}$, Pesoest fue $9 \pm 4$ vs. $7 \pm 3 \mathrm{cmH}_{2} \mathrm{O}$ $(p=0,2)$ y Pesodin, $6 \pm 2$ vs. $4 \pm 3 \mathrm{cmH}_{2} \mathrm{O}(\mathrm{p}=0,3)$, respectivamente. La correlación de Pesoest y din con PIAest fue 0,5 ( $p=0,003)$ y $0,4(p=0,03)$, respectivamente. Los componentes de la distensibilidad del sistema respiratorio estaban disminuidos (Csr, $31 \pm 8$; Cp, $52 \pm 22$; Cpt, $\left.105 \pm 50 \mathrm{ml} / \mathrm{cmH}_{2} \mathrm{O}\right)$, Cpt fue significativamente más baja en los pacientes con HIA (81 \pm 31 vs. $\left.118 \pm 55 \mathrm{ml} / \mathrm{cmH}_{2} \mathrm{O} ; \mathrm{p}=0,02\right)$. El coeficiente de correlación entre la PIAest y Cpt fue $-0,7$ $(\mathrm{p}<0,001)$ y de $-0,5(\mathrm{p}=0,002)$ con Csr.

Conclusiones: La pared torácica es más rígida en pacientes con hipertensión abdominal. En presencia de factores de riesgo de HIA las presiones en estos compartimentos son muy variables. (C) 2009 Elsevier España, S.L. y SEMICYUC. Todos los derechos reservados.
\end{abstract}

\footnotetext{
* Autor para correspondencia.

Correo electrónico: frferron@terra.es (F. Ruiz Ferrón).
} 


\section{KEYWORDS}

Intraabdominal

pressure;

Chest wall

compliance;

Esophageal pressure;

Mechanical

ventilation;

Abdominal

compliance
Intraabdominal and thoracic pressure in critically ill patients with suspected intraabdominal hypertension

\footnotetext{
Abstract

Objective: To study the correlation between intraabdominal and intrathoracic pressure in patients with suspected intraabdominal hypertension.

Design: A prospective, observational cohort study.

Setting: Polyvalent intensive care unit of a University hospital.

Patients: Twenty-seven medical-surgical patient dependent upon controlled mechanical ventilation due to acute respiratory failure and with several risk factors for intraabdominal hypertension (IAH).

Main variables: Intraabdominal (IAP), esophageal (Peso) and airways pressure were measured under static (st) and dynamic (dyn) conditions. Respiratory system (Crs), lung (Cl) and chest wall compliance $(\mathrm{CcW})$ were calculated.

Results: In 10 patients IAP > $12 \mathrm{mmHg}$ (IAH, IAPst, $14 \pm 2$ [12-21] mmHg), while in the rest the pressure proved normal $(\mathrm{n}=17$; IAPst, $8 \pm 2$ [3-11] $\mathrm{mmHg})$. Peso st was $11 \pm 5(2-27)$ and Peso dyn $7 \pm 4(2-24) \mathrm{cmH}_{2} \mathrm{O}$. Depending on the presence or absence of IAH, Peso st was $9 \pm 4$ vs $7 \pm 3 \mathrm{cmH}_{2} \mathrm{O}(\mathrm{p}=0.2)$ and Peso dyn $6 \pm 2$ vs $4 \pm 3 \mathrm{cmH}_{2} \mathrm{O}(\mathrm{p}=0.3)$, respectively. The correlation between Peso st and dyn with IAPst was $0.5(p=0.003)$ and $0.4(p=0.03)$, respectively. The compliance components were decreased ( $\mathrm{Crs}, 31 \pm 8 ; \mathrm{Cl}, 52 \pm 22$ and $\mathrm{CcW}, 105 \pm 50 \mathrm{ml} / \mathrm{cmH}_{2} \mathrm{O}$ ); $\mathrm{CcW}$ was significantly lower in patients with IAH $\left(81 \pm 31\right.$ vs $\left.118 \pm 55 \mathrm{ml} / \mathrm{cmH}_{2} \mathrm{O} ; \mathrm{p}=0.02\right)$. The correlation coefficient between IAPst and Ccw was $-0.7(p<0.001)$, and $-0.5(p=0.002)$ with respect to Crs.

Conclusions: A stiffer chest wall was observed in patients with IAH. In patients with risk factors for $\mathrm{IAH}$, pressures in these compartments were highly variable.

(c) 2009 Elsevier España, S.L. and SEMICYUC. All rights reserved.
}

\section{Introducción}

La reanimación masiva con líquidos, cirugía de abdomen, íleo, etc, produce hipertensión intraabdominal (HIA) en entre el 30 y el $80 \%$ de los pacientes críticos ${ }^{1}$; la incidencia es muy variable, así como el nivel de presión intraabdominal (PIA), que tiene implicaciones clínicas ${ }^{2}$. Por otro lado, la exploración clínica no es suficiente para determinar si hay o no $\mathrm{HIA}^{3}$ y se recomienda medir la presión intravesical ${ }^{4,5}$. Estos factores dificultan el manejo de estos pacientes, pero si no se considera el aumento de PIA, esta repercute sobre los demás compartimentos del organismo, favorece el síndrome policompartimental y el fallo multiorgánico ${ }^{1,6}$.

La transmisión de presión abdominal al compartimento torácico aumenta la rigidez del tórax ${ }^{7}$, comprime el pulmón ${ }^{8}$ y produce un aumento de la presión en la vía aérea. Determinar en un paciente con ventilación mecánica si las altas presiones en el respirador se deben a rigidez torácica o pulmonar no está al alcance de la exploración clínica, especialmente en casos de síndrome de distrés respiratorio agudo del adulto (SDRA) ${ }^{9}$. Para esto es necesario medir la presión abdominal y esofágica, que tiene, por lo tanto, implicaciones diagnósticas y terapéuticas. Permite diferenciar la rigidez torácica de la pulmonar, modificar los parámetros del respirador, especialmente del nivel de presión positiva al final de la espiración (PEEP), así como utilizar medidas para disminuir la presión abdominal. Por lo tanto, parece necesario valorar el efecto de la presión intraabdominal en el sistema respiratorio ${ }^{2}$. Sin embargo, en la práctica habitual no se mide la presión esofágica ${ }^{10}$, lo que se ha atribuido a la necesidad de un equipo adicional o de respiradores con sofisticados sistemas de monitorización y estas mediciones suelen ser utilizadas de forma aislada en estudios de investigación.

El objetivo de nuestro estudio es evaluar la correlación entre las presiones abdominal y torácica en pacientes con sospecha de hipertensión abdominal, sin utilizar dispositivos adicionales a los utilizados en pacientes críticos.

\section{Pacientes y métodos}

Estudiamos a 27 pacientes con insuficiencia respiratoria aguda en ventilación mecánica controlada, con sedación profunda (seis puntos en la escala de Ramsay) y, ocasionalmente, relajación muscular. Medimos la presión abdominal y esofágica por el riesgo de hipertensión abdominal y dificultades en la ventilación mecánica. El estudio fue autorizado por el comité ético del hospital y no se realizó ninguna intervención en el manejo habitual de estos pacientes.

\section{Presión intraabdominal (PIA)}

Las mediciones han sido realizadas siguiendo las recomendaciones de la reciente conferencia de consenso ${ }^{11}$. La presión intraperitoneal fue estimada a partir de la presión vesical medida con la sonda de Foley, conectada con una llave en $\mathrm{T}$ a una jeringa y con un transductor de presión (D85716, Edwards Lifesciencies, Unterschlessheim, Alemania), al monitor de cabecera (Marquette Hellige Solar 8000, Medical System, Milwaukee, Estados Unidos). La medición fue realizada en decúbito supino, con el transductor en la línea 
Tabla 1 Características de los pacientes

\begin{tabular}{lllll}
\hline & \multicolumn{4}{c}{ Diagnóstico } \\
\cline { 2 - 5 } & $\begin{array}{l}\text { Sepsis abdominal } \\
\text { no quirúrgica }\end{array}$ & $\begin{array}{l}\text { Peritonitis } \\
\text { secundaria }\end{array}$ & $\begin{array}{l}\text { Pancreatitis } \\
\text { aguda grave }\end{array}$ & $\begin{array}{l}\text { Parada } \\
\text { cardiorrespiratoria }\end{array}$ \\
\hline $\mathrm{n}$ & 4 & 12 & 5 & 6 \\
Edad (años) & $71 \pm 8$ & $67 \pm 11$ & $71 \pm 10$ & $68 \pm 12$ \\
Hombres & 2 & 4 & 4 & 2 \\
Apache II & $21 \pm 11$ & $21 \pm 4$ & $18 \pm 5$ & $27 \pm 10$ \\
Fallecimiento & 1 & 7 & 2 & 5 \\
\hline
\end{tabular}

axilar media y después de inyectar $20 \mathrm{ml}$ de suero salino $0,9 \%$. Confirmamos que el transductor mide correctamente con una ligera presión suprapúbica que produce una oscilación en la curva de presión. Con el cursor del monitor medimos la presión al final de la espiración o estática (PIAest) y la oscilación respiratoria o presión dinámica (PIAdin), estimador de la distensibilidad del abdomen ${ }^{12}$, como la diferencia entre el final de la inspiración y la espiración. Obtenemos el valor medio de al menos tres mediciones con una variabilidad menor del $10 \%$.

\section{Presiones intratorácicas}

La presión esofágica fue medida con la sonda nasogástrica que tiene el paciente para nutrición o drenaje gástrico (tipo Levin $125 \mathrm{~cm}, 14-16 \mathrm{~F}$, Unomedical, Dinamarca), sin equipos adicionales ${ }^{13}$. Medimos la presión transmitida por líquido ${ }^{14}$, de forma similar a la técnica de medida de la presión vesical, la sonda es conectada con una llave en $\mathrm{T}$ a una jeringa y un transductor. Por ser de material plástico transparente, permite observar si hay burbujas $u$ otro material en su interior. Este método ha sido validado en estudios experimentales ${ }^{15}$ y clínicos $^{14,16}$. Para confirmar la posición de la sonda en el esófago no podemos realizar un test de oclusión por la ausencia de esfuerzos respiratorios. Para esto comprobamos en la radiología de tórax la posición del extremo distal y con una ligera compresión epigástrica confirmamos su posición. Retiramos lentamente hasta observar el cambio de presión y la transmisión del latido cardiaco, la primera señal de la sonda $(37 \mathrm{~cm})$ siempre se visualiza ${ }^{17}$. Previamente a un nuevo purgado con salino, aspiramos suavemente para retirar aire o líquido retenidos en el esófago. Las mediciones son realizadas con el cabecero del paciente elevado y el transductor en la línea axilar media. Cuando la curva de presión tiene un registro estable, realizamos una prueba de lavado rápido del transductor, comprobamos la respuesta al aumento de presión y la recuperación de la presión previa. Con el cursor del monitor medimos la presión al final de la espiración o estática (Pesoest) y la oscilación respiratoria o presión dinámica (Pesodin), como la diferencia entre el final de la inspiración y la espiración. Utilizamos como referencia un punto medio de la oscilación del latido cardiaco. Obtenemos el valor medio de al menos tres mediciones con una variabilidad menor del $10 \%$.

La distensibilidad del sistema respiratorio (Csr), pared torácica (Cpt) y pulmonar (Cp) fueron medidas como la relación entre el volumen corriente y el delta de presión en la vía aérea (presión meseta - PEEP total), delta de presión esofágica (cambio de presión esofágica entre final de la inspiración y la espiración) y el delta de presión en la vía aérea menos el delta de presión esofágica, respectivamente. Con el respirador en modo controlado por volumen con flujo constante y una pausa inspiratoria de 0,1-0,2 s. Las presiones de la vía aérea han sido medidas en la pantalla del respirador en $\mathrm{CmH}_{2} \mathrm{O}$ y las medidas en el monitor en $\mathrm{mmHg}$ son transformadas en $\mathrm{cmH}_{2} \mathrm{O}$ $\left(1 \mathrm{mmHg}=1,36 \mathrm{cmH}_{2} \mathrm{O}\right)$.

\section{Análisis estadístico}

Comprobamos que las variables cuantitativas estudiadas siguen una distribución normal mediante el test de Kolmogorov-Smirnov. Los resultados descriptivos de las variables cuantitativas son expresados como media \pm desviación estándar, en las variables de interés mostramos el intervalo. La comparación de medias entre los pacientes con y sin hipertensión abdominal se realizó mediante la t de Student. La correlación entre las variables cuantitativas (Pesoest, Pesodin, PIAest, PIAdin), con el test de Pearson. Consideramos como estadísticamente significativo un valor de $p<0,05$.

\section{Resultados}

Las características de los pacientes y los parámetros de ventilación los mostramos en las tablas 1 y 2 . En 10 de los 27 pacientes la PIAest fue mayor de $12 \mathrm{mmHg}(37 \%)$. El coeficiente de correlación entre la presión intraabdominal estática (PIAest, $10 \pm 3$ [3-21] mmHg) y dinámica (PIAdin,

\section{Tabla 2 Parámetros de ventilación}

Vc $(\mathrm{ml})$ $582 \pm 55(480-700)$

$\mathrm{Fr}(\mathrm{rpm})$

$16 \pm 2(10-22)$

V'I $(\mathrm{l} / \mathrm{m})$

$42 \pm 6(33-60)$

$\mathrm{Pi}_{\text {máx }}\left(\mathrm{cmH}_{2} \mathrm{O}\right)$

$38 \pm 9(27-72)$

$\mathrm{Pm}\left(\mathrm{cmH}_{2} \mathrm{O}\right)$

$\mathrm{FIO}_{2}(\%)$

PEEP $\left(\mathrm{cmH}_{2} \mathrm{O}\right)$

$26 \pm 8(18-55)$

$55 \pm 15(40-100)$

$6 \pm 2(3-12)$

$\mathrm{FiO}_{2}$ : fracción inspirada de oxígeno; FR: frecuencia respiratoria; $\mathrm{Pi}_{\text {máx }}$ : presión inspiratoria máxima; $\mathrm{PEEP}$ : presión positiva al final de la espiración; Pm: presión meseta; Vc: volumen corriente; V'l: flujo inspiratorio.

Los datos expresan media \pm desviación estándar (intervalo). 


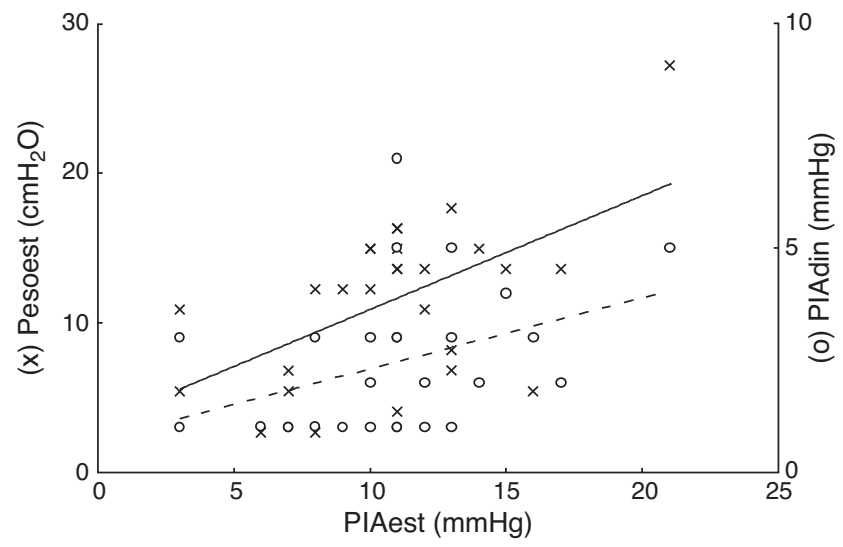

Figura 1 Relación entre la presión intraabdominal estática (PIAest, eje $\mathrm{x}$ ) y la presión esofágica estática (Pesoest, eje y izquierdo, circulos en negro). Línea continua ajuste a regresión lineal, Pesoest, 3,2 \pm 0,7.PIAest y relación con la presión intraabdominal dinámica (PIAdin, eje y derecho, círculos en blanco), ajuste en línea discontinua; PIAdin, 0,7 $\pm 0,15$.PIAest. El aumento de presión abdominal se relaciona con la rigidez torácica y abdominal, pero hay una amplia variabilidad entre pacientes, que posiblemente cause la débil correlación. En la práctica, dentro del rango de presiones estudiado, cuando la palpación del abdomen es anormal (PIAdin, estimador de la distensibilidad abdominal), no podemos inferir el cambio en la presión intraabdominal ni intratorácica.

$2,4 \pm 1,6[1-7])$ fue $0,4(p=0,04), 0,5$ en el grupo con hipertensión abdominal (PIAest, $14 \pm 2$ [12-21] $\mathrm{mmHg}$ ) y de 0,3 en los pacientes sin HIA ( $n=17$; PIAest, $8 \pm 2$ [3-11] $\mathrm{mmHg}$ ) (fig. 1). La PIAdin fue independiente de la presencia o no de HIA (sin HIA, 2,2 $\pm 1,7$ [1-7] mmHg vs. HIA, 2,8 $\pm 1,4$ [1-5] $\mathrm{mmHg} ; \mathrm{p}=0,4)$. La presión esofágica estática fue $11 \pm 5(2$ 27) $\mathrm{cmH}_{2} \mathrm{O}$ y la dinámica, $7 \pm 4(2-24) \mathrm{cmH}_{2} \mathrm{O}$. Considerando la presencia o no de $\mathrm{HIA}$, Pesoest fue de $9 \pm 4 \mathrm{vs.} 7 \pm 3 \mathrm{cmH}_{2} \mathrm{O}$ $(p=0,2)$ y Pesodin, $6 \pm 2$ vs. $4 \pm 3 \mathrm{cmH}_{2} \mathrm{O}(\mathrm{p}=0,3)$, respectivamente. La correlación de la presión abdominal estática $\left(11 \pm 5[2-27] \mathrm{cmH}_{2} \mathrm{O}\right)$ con la presión esofágica fue de 0,5 $(p=0,003)$ y de $0,4(p=0,03)$ con Pesodin. La correlación de la PIAest con la distensibilidad de la pared torácica fue de $-0,69(p<0,001)$ y de $-0,56(p=0,002)$ con la distensibilidad del sistema respiratorio. Las distensibilidades del sistema respiratorio, pulmonar y torácico estaban disminuidas $\left(31 \pm 8,52 \pm 22,105 \pm 50 \mathrm{ml} / \mathrm{cmH}_{2} \mathrm{O}\right.$, respectivamente). El coeficiente de correlación entre PIAest y Cpt utilizando una ecuación exponencial $\left(y=a x^{b}\right)$ aumenta $a-0,81$ (fig. 2), no se modifica entre PIAest y la presión esofágica estática o dinámica. En los pacientes con HIA la distensibilidad de la pared torácica fue significativamente menor que en los pacientes sin HIA ( $81 \pm 31$ vs. $\left.118 \pm 55 \mathrm{ml} / \mathrm{cmH}_{2} \mathrm{O} ; \mathrm{p}=0,02\right)$. Las distensibilidades del sistema respiratorio y pulmonar no fueron significativamente inferiores (fig. 3) (Csr, $28 \pm 9$ vs. $33 \pm 7 \mathrm{cmH}_{2} \mathrm{O}$ y $\mathrm{Cp}, 50 \pm 21$ vs. $54 \pm 23$ ). La presión esofágica estática no se correlaciona con la distensibilidad de la pared torácica $(r=0,01)$.

En seis pacientes que recibían ventilación con PEEP $\geq$ $10 \mathrm{cmH}_{2} \mathrm{O}$ las presiones en el abdomen y el tórax tienden a ser más altas, pero no de forma significativa respecto a los paciente con PEEP menor de $10 \mathrm{cmH}_{2} \mathrm{O}$ (PIAest $13 \pm 3$ vs. $10 \pm 3 \mathrm{mmHg}$; Pesoest $9 \pm 2$ vs. $8 \pm 4 \mathrm{mmHg}$ ).

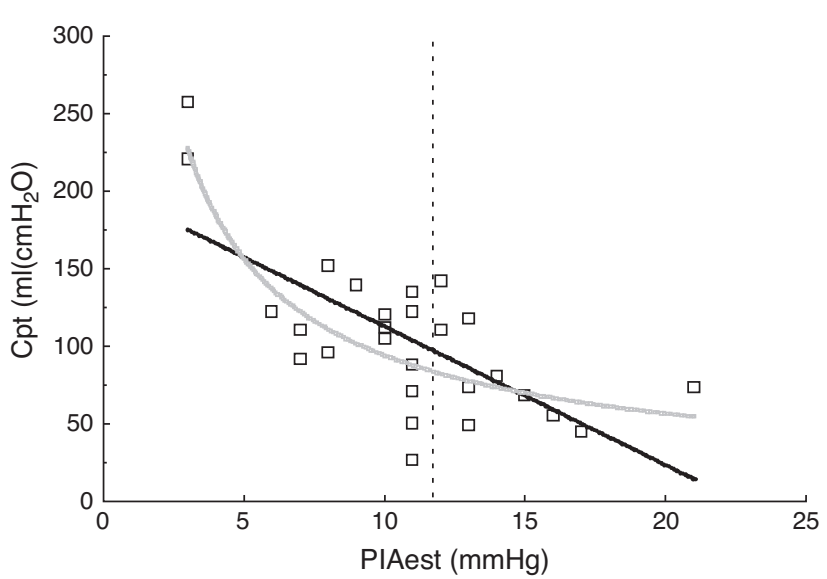

Figura 2 Eje x. Presión intraabdominal estática $(\mathrm{mmHg})$. Eje $\mathrm{y}$, distensibilidad de la pared torácica $\left(\mathrm{ml} / \mathrm{cmH}_{2} \mathrm{O}\right)$. Línea negra, ajuste a un modelo lineal $(\mathrm{Cpt}=-8,92-\mathrm{PIAest}+201)$. Línea gris, ajuste a un modelo exponencial $\left(\mathrm{Cpt}=510\right.$-PIAest $\left.{ }^{-0,73}\right)$. Línea discontinua, PIAest, $12 \mathrm{mmHg}$ (HIA).

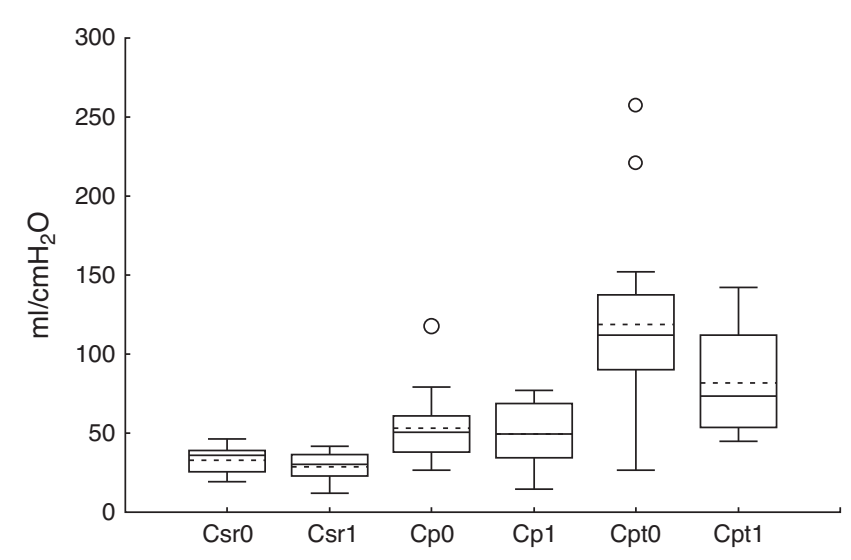

Figura 3 Gráfico box-plot. Eje y. Distensibilidad: $\mathrm{ml} / \mathrm{cmH}_{2} \mathrm{O}$. Eje x. Componentes de la distensibilidad: Csr, Cp, Cpt. Distensibilidad del sistema respiratorio, pulmonar y pared torácica. 0 , 1 , sin y con hipertensión abdominal, respectivamente.

\section{Discusión}

Los resultados de nuestro estudio demuestran que en pacientes con factores de riesgo de hipertensión abdominal las presiones intraabdominal e intratorácica son muy variables. En un tercio de los casos encontramos hipertensión abdominal, grupo de pacientes en el que las presiones intratorácicas son más altas y, sobre todo, tienen una mayor rigidez torácica.

La presión vesical es el estándar ${ }^{5}$ para estimar la PIA, pero es necesario que el contenido del abdomen actúe como un único compartimento para que la presión vesical refleje la PIA ${ }^{7,18}$. De lo contrario, la presión intraorgánica (vesical, gástrica, etc.) será variable ${ }^{7,18}$ y la presión vesical puede no reflejar la presión intraperitoneal ${ }^{19}$. La PIA que medimos fue más alta que la referida en pacientes con ventilación mecánica $(5-7 \mathrm{mmHg})^{11}$, pero similar a la descrita cuando hay factores de riesgo de HIA $\left(14 \pm 1^{20}, 11 \pm 0^{21} \mathrm{mmHg}\right)$ o HIA $\left(15 \pm 3 \mathrm{mmHg}^{1,22}\right)$. Estos valores más altos de PIA facilitan que el abdomen tenga una mecánica hidráulica y la presión 
intravesical refleje la presión intraperitoneal ${ }^{6}$. Encontramos hipertensión abdominal en, aproximadamente, un tercio de los pacientes estudiados y los valores medidos fueron muy variables de unos pacientes a otros. Esta dispersión de valores ha sido descrita en otros estudios, en pacientes con afección médica $(8-36 \mathrm{mmHg})^{1,3,6}$, quirúrgica y en politraumatizados $(2-94 \mathrm{mmHg})^{23}$. Además, coincidiendo con estudios previos ${ }^{21}$, la distensión del abdomen objetivada por la PIAdin mostró una débil correlación con la presión abdominal (PIAest). Estos factores indican que en pacientes con factores de riesgo de HIA es necesario medir la PIA, ya que la valoración clínica es insuficiente, al menos dentro del rango de presiones que mostraban los pacientes estudiados.

La presión esofágica al final de la espiración se ha utilizado poco para estimar la presión pleural, principalmente porque el peso del mediastino influye en esta medida. Pero se ha demostrado que este artefacto representa menos de $5 \mathrm{cmH}_{2} \mathrm{O}^{24}$, que representa un pequeño porcentaje dentro del rango de presión esofágica estática descrita en estos pacientes ${ }^{2}$. Así, los valores que obtuvimos son similares a los referidos en pacientes con SDRA $\left(10-12 \mathrm{cmH}_{2} \mathrm{O}\right)^{25}$, aunque también se han descrito valores más altos $\left(17 \pm 5 \mathrm{cmH}_{2} \mathrm{O}\right)^{10}$. Por el contrario, con respiración espontánea en posición supina la presión es más baja $\left(0,7-5,3 \mathrm{cmH}_{2} \mathrm{O}\right)^{26}$. Esta diferencia no se debe a la ventilación mecánica, pues esta no cambia la mecánica de la caja torácica ${ }^{27}$ y se han referido valores de $-0,8 \pm 1,9 \mathrm{cmH}_{2} \mathrm{O}^{28}$. Pero en pacientes críticos con respirador, múltiples factores cambian la mecánica de la caja torácica (obesidad, edema, cirugía abdominal, etc.) y el rango de la presión esofágica es muy amplio e impredecible $\left(4-32 \mathrm{cmH}_{2} \mathrm{O}^{2}\right)$.

Con más frecuencia se utiliza la variación respiratoria de la presión esofágica (Pesodin) para valorar la distensibilidad del tórax ${ }^{29}$. Los valores que obtuvimos fueron más altos que los previamente referidos en pacientes con fallo respiratorio agudo $\left(4 \pm 4 \mathrm{cmH}_{2} \mathrm{O}\right)^{10}$ y de acuerdo con otros estudios encontramos que el aumento de PIA produce un aumento en la rigidez de la pared torácica ${ }^{17,29}$. Así Gattinoni et $\mathrm{al}^{30}$ refieren un coeficiente de correlación de 0,84 en pacientes con valores de PIA de $5-35 \mathrm{mmHg}$. Pero también se han descrito resultados opuestos, sin correlación entre la distensibilidad torácica y la PIA con valores de $16 \pm 3$ y $19,3 \pm 7,8 \mathrm{mmHg}^{17,31}$. Esta discrepancia se ha atribuido al diferente rango de PIA que presentaban los pacientes de estos estudios, pero posiblemente intervienen otros factores ${ }^{32}$. Fundamentalmente, la distensibilidad de la pared abdominal ${ }^{33}$, torácica ${ }^{34}$ y pulmonar ${ }^{35}$ que amortiguan los cambios de presión torácica hasta los $20 \mathrm{mmHg}$ de $\mathrm{PIA}^{31,35}$. De este modo, la transmisión de PIA al tórax puede variar entre el 25 y el $80 \%^{36,37}$, lo que indica que la interacción mecánica entre el abdomen y el tórax es compleja ${ }^{38}$. De acuerdo con esto, nuestros resultados muestran que un modelo exponencial se ajusta mejor a la relación entre presión abdominal y distensibilidad torácica.

Medimos la distensibilidad respiratoria sin interrumpir la ventilación, por tanto en la presión elástica hay un componente resistivo, debido principalmente a la viscoelasticidad del tórax. Pero este es poco importante, como se ha demostrado en pacientes con obesidad mórbida $^{39}$. Los componentes de la distensibilidad del sistema respiratorio que medimos son similares a los descritos en estos pacientes ${ }^{40}$. La distensibilidad de la pared torácica fue un $37 \%$ menor que en pacientes anestesiados $\left(105 \pm 50 \mathrm{ml} / \mathrm{cmH}_{2} \mathrm{O} \text { vs. } 167 \mathrm{ml} / \mathrm{cmH}_{2} \mathrm{O} ; \mathrm{p}=0,001\right)^{41}$, por lo tanto, la distensibilidad del sistema respiratorio subestima a la distensibilidad pulmonar. La distensibilidad torácica no se correlacionó con la presión esofágica estática, esto ha sido referido en otros estudios ${ }^{2,17}$ y se ha atribuido a que la distensibilidad torácica está influida por el volumen de ventilación y la distensibilidad del sistema respiratorio ${ }^{42}$. Pero en este tipo de pacientes, posiblemente el principal factor es el peso del abdomen sobre el esófago, puede aumentar la presión esofágica estática sin modificar la distensibilidad torácica ${ }^{43}$, como se ha demostrado en sujetos con obesidad mórbida ${ }^{44}$. En nuestro estudio puede influir el método que utilizamos para medir la presión esofágica, con catéter lleno de suero. Aunque este método ha sido previamente utilizado en estudios clínicos ${ }^{45}$, puede haber un artefacto cuando hay un gradiente hidrostático de presión entre el extremo distal del catéter y el transductor ${ }^{46}$. Esto no influye en el método de referencia de medida de la presión esofágica (catéter con balón $)^{47}$, pero tampoco este método es exacto, debido a la posición y el volumen del balón ${ }^{48}$.

En resumen, los resultados de nuestro estudio indican que en pacientes con factores de riesgo de hipertensión abdominal es necesario medir la presión abdominal, ya que la valoración clínica es insuficiente y estos pacientes presentan un descenso de la distensibilidad torácica. La medida de la presión esofágica permite valorar más adecuadamente la mecánica respiratoria y posiblemente optimizar la ventilación mecánica en pacientes con hipertensión abdominal.

\section{Bibliografía}

1. Malbrain ML, Chiumello D, Pelosi P, Bihari D, Innes R, Ranieri VM, et al. Incidence and prognosis of intraabdominal hypertension in a mixed population of critically ill patients: A multiple-center epidemiological study. Crit Care Med. 2005;33:315-22.

2. Talmor D, Sarge T, O’Donnell C, Ritz R, Malhorta A, Lisbon A, et al. Esophageal and transpulmonary pressure in acute respiratory failure. Crit Care Med. 2006;34:1389-94.

3. Sugrue M, Bauman A, Jones F, Bishop G, Flabouris A, Parr M, et al. Clinical examination is an inaccurate predictor of intraabdominal pressure. World J Surg. 2002;26:1428-31.

4. Gudmundsson F, Viste A, Gislason H, Svanes K. Comparison of different methods for measuring intra-abdominal pressure. Intensive Care Med. 2002;28:509-14.

5. Kron IL, Hartman PK, Nolan SP. The measurement of intraabdominal pressure as a criterion for abdominal re-exploration. Ann Surg. 1984;199:28-30.

6. Malbrain ML. Abdominal pressure in the critically ill: measurement and clinical relevance. Intensive Care Med. 1999;25:1453-8.

7. Obeid F, Saba A, That J, Buslits B, Chung R, Sorensen V, et al. Increases in intrabdominal pressure affects pulmonary compliance. Arch Surg. 1995;130:544-8.

8. Gattinoni L, Vagginelli F, Chiumello D, Taccone P, Carlesso E. Physiologic rationale for ventilator setting in acute lung injury/acute respiratory distress syndrome patients. Crit Care Med. 2003;31:S300-4.

9. Chiumello D, Carlesso E, Cadringgher P, Caironi P, Valenza $F$, Polli $F$, et al. Lung stress and strain during mechanical ventilation for acute respiratory distress syndrome. Am J Respir Crit Care Med. 2008;178:346-55. 
10. Talmor D, Sarge T, Malhotra A, O’Donnell CR, Ritz R, Lisbon A, et al. Mechanical ventilation guided by esophageal pressure in acute lung injury. N Engl J Med. 2008;359:2095-104.

11. Malbrain ML, De laet I, Cheatham M. Consensus conference definition and recommendations on intra-abdominal hypertension (IAH) and the abdominal compartment syndrome (ACS): the long road to the final publications, how did we get there. Acta Clin Belg. 2007;62 Suppl 1:44-59.

12. Sturini E, Saporito A, Sugrue M, Parr M, Bishop G. Respiratory variation in intra-abdominal pressure $(\triangle I A P)$. Acta Clin Belg. 2007;62 Suppl 1:265.

13. Panizza JA, Finucane KE. Comparison of ballon and transducer catheters for estimating lung elasticity. J Appl Physiol. 1992;72:231-5.

14. Giannouli E, Webster K, Roberts D, Younes M. Response of ventilator dependent patients to different levels of pressure support and proportional assist. Am J Respir Crit Care Med. 1999;159:1716-25.

15. Ask P, Oberg A, Tibbling L. Static and dynamic characteristics of fluid-filled esophageal manometry systems. Am J Physiol. 1977;233:E389-96.

16. Asher MI, Coates AL, Collinge JM, Milic-Emili J. Measurement of pleural pressure in neonates. J Appl Physiol. 1982;52:491-4.

17. Loring S, O'Donnell C, Behazin N, Malhotra A, Sarge T, Ritz $\mathrm{R}$, et al. Esophageal pressures in acute lung injury: do they represent artifact or useful information about transpulmonary pressure, chest wall mechanics, and lung stress? J Appl Physiol. 2010;108:515-22.

18. Shay S, Schreiber M, Richter J. Compliance curves during peritoneal dialysate infusión are like a distensible tube and are similar at multiple UGI sites. Am J Gastr. 1999;94:1034-40.

19. Olofsson PH, Berg S, Henrik CA, Brudin LH, Vikstrom T, Johansson KJM. Gastrointestinal microcirculation and cardiopulmonary function during experimentally increased intra-abdominal pressure. Crit Care Med. 2009;37:230-9.

20. Regueira T, Bruhn A, Hasbun P, Aguirre M, Romero C, Llanos O, et al. Intra-abdominal hipertensión: indicence and association with organ dysfunction during early septic shock. J Crit Care. 2008;23:461-7.

21. Sturini E, Saporio A, Sugrue M, Parr M, Bishop G, Braschi A. Respiratory variation of intra-abdominal pressure: indirect indicador of abdominal compliance? Intensive Care Med. 2008;34:1632-7.

22. Malbrain MLNG. Relationship of body mass index (BMI), lactate and intraabdominal pressure (IAP) to subsequent mortality in ICU patients. Crit Care Med. 1999;3 Suppl 1:P039.

23. Cheatham ML, White MW, Sagraves SG, Johnson JL, Block EFJ. Abdominal perfusion pressure: a superior parameter in the assessment of intra-abdominal hypertension. J Trauma. 2000;4:621-7.

24. Washko GR, O'Donnell CR, Loring SH. Volume-related and volume-independent effect of posture on esophageal and transpulmonary pressure in healthy subject. J Appl Physiol. 2006;100:753-8.

25. Mergoni M, Martelli A, Volpi A, Primavera S, Zuccoli P, Rossi A. Impact of positive end-expiratory pressure on chest wall and lung pressure-volume curve in acute respiratory failure. Am J Respir Crit Care Med. 1997;156:846-54.

26. Mead J, Gaensler EA. Esophageal and pleural pressures in man, upright and supine. J Appl Physiol. 1959;14:81-3.

27. Rehder K, Marsh HM. Respiratory mechanics during anesthesia and mechanical ventilation. En: WO, Fenn, $\mathrm{H}$, Rahn, editores. Handbook of physiology, sección 3: The respiratory system, Vol III: Mechanics of breathing, part, 2. Washington DC: Am Physiol, 1986. p. 737-52.

28. Jardin F, Farcot JC, Boisante L, Curien N, Margairaz A, Bourdarias JP. Influence of positive end-expiratory pressure on left ventricular performance. N Engl J Med. 1981;304:387-92.
29. Ranieri VM, Brienza N, Santostasi S, Puntillo F, Mascia L, Vitale $\mathrm{N}$, et al. Impairement of lung and chest wall mechanics in patients with acute respiratory distress syndrome. Role of abdominal distension. Am J Respir Crit Care Med. 1997; 156:1082-91.

30. Gattinoni L, Pelosi P, Suter PM, Pedoto A, Vercesi P, Lissoni A. Acute respiratory distress syndrome caused by pulmonary and extrapulmonary disease: Different syndromes? Am J Respir Crit Care Med. 1998;158:3-11.

31. Krebs J, Pelosi P, Tsagogiorgas C, Alb M, Luecke T. Effects of positive end-expiratory pressure on respiratory function and hemodynamics in patients with acute respiratory failure with and without intra-abdominal hipertensión: a pilot study. Crit Care. 2009;13:1-11.

32. Gilroy RJ, Lavietes MH, Loring SH, Mangura BT, Mead J. Respiratory mechanical effects of abdominal distension. J Appl Physiol. 1985;58:1997-2003.

33. Mutoh T, Lamm WJE, Embree LJ, Hildebrandt J, Albert RK. Abdominal distensión alters regional pleural pressures and chest mechanics in pigs in vivo. J Appl Physiol. 1991;70:2611-8.

34. O'Quin R, Marini JJ, Culver BH, Butler J. Transmisión of airway pressure to pleural space during edema and chest wall restriction. J Appl Physiol. 1985;59:1171-7.

35. Quintel M, Pelosi P, Caironi P, Meinhardt P, Luecke T, Herrmann $P$, et al. An increase of abdominal pressure increases pulmonary edema in oleic acid-induced lung injury. Am J Respir Crit Care Med. 2004;169:534-41.

36. Malbrain ML, Wilmer A. The polycompartment síndrome: Towards an understanding of the interactions between different compartments! Intensive Care Med. 2007;33:1869-72.

37. Malbrain MLNG, Deeren D, De Potter JR. Intra-abdominal hypertension in the critically ill: it is time to pay attention. Curr Opinion Crit Care. 2005;11:156-71.

38. Richardson JD, Trinkle JK. Hemodynamic and respiratory alterations with increased intra-abdominal pressure. J Surg Res. 1976;20:401-4.

39. Pelosi P, Croci M, Ravagnan I, Vicardi P, Gattinoni L. Total respiratory system, lung, and chest wall mechanics in sedatedparalyzed postoperative morbidly obese patients. Chest. 1996;109:144-51.

40. Katz JA, Zinn SE, Ozanne GM, Fairley HB. Pulmonary, chest wall and lung-thorax elastances in acute respiratory failure. Chest. 1981;80:304-11.

41. Grimby G, Hedenstierna G, Lofstrom B. Chest wall mechanics during artificial ventilation. J Appl Physiol. 1975;38: 576-80.

42. Agostoni E, Hyatt RE. Static behaviour of the respiratory system. En: Geiger SR, editor. Handbook of Physiology, Section 3: The Respiratory System. Part 1. III. Bethesda, MD: American Physiological Society; 1986. p. 113-30.

43. Hubmayr RD. Is there a place for esophageal manometry in the care of patients with injured lungs? J Appl Physiol. 2010;108:481-2.

44. Suratt PM, Wilhoit SC, HsiaoHS, Atkinson RL, Rochester DF. Compliance of chest wall in obese subjects. J Appl Physiol. 1984;57:403-7.

45. Kimura T, Takezawa J, Nishiwaki K, Shimada Y. Determination of the optimal pressure support level evaluated by measuring transdiaphragmatic pressure. Chest. 1991;100:112-7.

46. Mead J, Mcllroy MB, Selverstone NJ, Kriete BC. Measurement of intraesophageal pressure. J Appl Physiol. 1955;7:491-5.

47. Hartford CG, Rogers GC, Turner MJ. Correctly selecting a liquid-filled nasogastric infant feeding catéter to measure intraesophageal pressure. Pediatr Pulmonol. 1997;23:362-9.

48. Drummond GB, Wright AD. Inaccurate of oesophageal pressure for pleural pressure estimation in supine anaesthetized subjects. Br J Anaesth. 1983;55:585-93. 